\title{
Observation at Moshiri $(L=1.6)$ of Whistler-Triggered VLF Emissions in the Electron Slot and Inner Radiation Belt Regions
}

\author{
Masashi HAYAKAWA \\ Solar-Terrestrial Environment Laboratory, Nagoya University, Toyokawa, Aichi 442, Japan
}

(Received September 12, 1990; Revised December 17, 1990)

\begin{abstract}
The morphological characteristics of whistler-triggered VLF emissions including diurnal, seasonal variations, $K p$ dependence, latitudinal distribution and spectral shape, were investigated based on the VLF data obtained at Moshiri $(L=1.6)$ in Japan during a ten year span (1976 to 1985). The following results emerged; (1) There is no clear tendency for whistler-triggered emissions to occur at a particular local time, (2) an equinoctial maximum in occurrence probability is recognized, (3) the occurrence probability increases with increasing $K p$ in the range from 3 to 7 , (4) the occurrence $L$ shell is localized in two regions; one is $L=2.1$ to 3.4 (the electron slot region) and the other is just around $L=1.6$ (the inner radiation belt), and (5) a whistler-triggered emission is characterized by an initial component quasi-constant frequency and a subsequent large frequency drift with $\mathrm{d} f / \mathrm{d} t=10-20 \mathrm{kHz} / \mathrm{s}$. These characteristics are satisfactorily interpreted in terms of the gyroresonance interaction between lightning-generated whistlers and energetic electrons, with reference to previous results on lightning-induced particle precipitation.
\end{abstract}

\section{Introduction}

The gyroresonance interaction between whistler-mode waves and energetic electrons is an important process in the magnetosphere. Its consequences are the linear wave amplification and generation of VLF emissions, and also the wave-induced pitch angle scattering of magnetospheric particles and associated particle precipitation into the lower ionosphere (e.g. BRICE, 1964).

Lightning-generated whistlers have been recently found to induce particle precipitation (HELliWELl et al., 1973; CARPENTER and LABELLE, 1982; LEYSER et al., 1984; VOSS et al., 1984; INAN et al., 1985; INAN and CARPENTER, 1987; CARPENTER and INAN, 1987), but much remains to be learned concerning the contributions to the loss of radiation belt electrons from magnetospheric waves of different origins such as hiss, chorus, whistlers, VLF transmitter signals etc. So, in order to investigate the role of lightning-generated whistlers in the magnetospheric wave-particle interaction process and in the removal of energetic electrons from the radiation belts, we studied the general characteristics of VLF emissions triggered by lightning-generated whistlers as a result of strong nonlinear gyroresonance interaction. The presence of such whistler-triggered VLF emissions has long been known from the beginning of whistler studies (HELLIWELL, 1965), but no reports have been published on the statistical features of these whistlertriggered VLF emissions based on ground-based VLF data. Hence, the present paper is the first attempt to examine the statistical characteristics of whistler-triggered VLF emissions including diurnal and seasonal variations, $K p$ dependence, latitudinal distribu- 
tion and spectral shape, on the basis of VLF data observed at Moshiri ( $L=1.6)$, Japan during a ten year span, from 1976 to 1985 . Lightning-induced particle precipitation which is the particle aspect of the gyroresonance interaction has been lately investigated by many authors (HELLIWELL et al., 1973; CARPENTER and LABELLE, 1982; LEYSER et al., 1984; VOSS et al., 1984; INAN et al., 1985, 1988; INAN and CARPENTER, 1987; CARPENTER and INAN, 1987). So, using these previous studies of lightning-induced particle precipitation as a reference, we discuss the general characteristics of whistlertriggered emissions from the point of view of gyroresonance interaction.

\section{VLF Data Base}

The present study is based on VLF observations made at Moshiri (Inv. lat. $38^{\circ} \mathrm{N}$; $L=1.6$ ), Japan (HAYAKAWA et al., 1975, 1985; HAYAKAWA, 1989) during ten years, from 1976 to 1985 . VLF wideband signals (up to $10 \mathrm{kHz}$ ) were recorded on magnetic tapes on a routine basis (50-52 minutes every hour) during this period of ten years. We made an aural monitoring of the recorded tapes and when we aurally found any VLF/ELF emissions, we displayed them as sonagrams. After a close inspection of all sonagrams of VLF/ELF emissions, we could find 74 events definitely identified as VLF emissions triggered by whistlers. The requirement for a given event to be considered as a whistlertriggered VLF emission, was that its initial portion was originated in the spectrum of the causative whistler. We found that triggered emission events mainly take place on different days, but some occur on the same day, but at a different time. Thus, statistical characteristics in the following sections are considered to be meaningful, even with such a small data set of 74 events.

\section{Characteristics of Whistler-Triggered Emissions}

We present the information concerning the occurrence of whistler-triggered VLF emission events, with emphasis on local time, seasonal and $K p$ dependencies, path latitude distribution and spectral properties. The full line in Fig. 1(a) indicates the relationship between the occurrence number of whistler-triggered VLF emissions and U.T. $($ L.T. $=$ U.T. $+9 \mathrm{~h}$ ), and the broken line refers to the total occurrence number of whistlers recorded during the studied ten years. The broken line indicates that the diurnal variation of total occurrence number of whistlers exhibits two maxima; a major broad one at L.T. $=17 \mathrm{~h}$ and a secondary broad one at L.T. $=3 \mathrm{~h}$. This pattern is consistent with the previous result from Wakkanai (very close to Moshiri) by KIMPARA (1961). However, when the latitude of the observing station becomes higher, such as $L>2$, the whistler occurrence rate exhibits only a single peak between midnight and dawn (HELLIWELL, 1965). The comparison of values given by the full and broken lines yields the occurrence probability of whistler-triggered VLF emissions, and there does not seem to be a clear tendency for whistler-triggered emissions to occur at a particular L.T. So, the whistler-triggered emissions are found to occur over the whole L.T. though a small enhancement is seen at L.T. $=14 \mathrm{~h}$.

Figure 1(b) illustrates the seasonal variation of whistler-triggered VLF emissions in a full line, while a broken line indicates the corresponding seasonal variation of the total whistlers recorded. The broken line indicates that whistler occurrence rate at medium latitudes exhibits a maximum in winter (December, January, and February). This is in 
agreement with the previous studies by KIMPARA (1960), HELLIWELL (1965) and HAYAKAWA et al. (1971). The occurrence probability of whistler-triggered emissions is given by the ratio of the values indicated by full and broken lines, as it is indicated by dots in the figure. The occurrence probability exhibits enhancements in March and in September-October, and equinoctial occurrence is likely to be an important characteristic of whistler-triggered emissions.

Figure 1(c) illustrates the occurrence histogram of whistler-triggered VLF emission events versus $K p$ index at the time of emission observation in a full line, while a broken line indicates the percentage occurrence of number of times $K p$ is in each range during the ten-year span. The occurrence probability of whistler-triggered emissions as based on the values indicated by the full and broken lines, is indicted by a dot in the figure. The occurrence probability is found to be rather small in the $K p$ range from 0 to 2, and then we notice a general tendency for the occurrence probability to increase with increasing $K p$ in the range from 3 to 7 . This increase in occurrence probability in this $K p$ range implies that the whistler-triggered emissions are strongly correlated with geomagnetic activity such as to occur during moderate and severe geomagnetic disturbances. This $K p$ dependence of whistler-triggered emissions is in sharp contrast with the corresponding $K p$ dependence of whistler occurrence numbers studied by ALLCOCK (1966) who showed that the whistler activity reaches a maximum at $K p=4$ at $L=2.0$.

In Fig. 2 we present an example of VLF emissions triggered by whistlers. In the figure we find a group of whistlers, a whistler with dispersion of $50 \mathrm{sec}^{1 / 2}$ nearly at the end of the group appears to trigger a discrete VLF emission with dynamic frequency variation. At the lowest end $(\sim 2 \mathrm{kHz})$ of the whistler spectrum, we notice a quasiconstant frequency weak component, followed by a sharp frequency increase and a subsequent small frequency increase. Figure 3 summarizes the spectral properties of whistler-triggered emissions including the one in Fig. 2. In producing Fig. 3 we concentrated on about 30 obvious events to obtain definite information. The second panel in Fig. 3 illustrates the local time variation of the frequency range occupied by triggered VLF emissions so the lowest tip of each bar indicates nearly the starting frequency of discrete VLF emissions. The top panel in Fig. 3 illustrates the local time variation of frequency drift of triggered emissions, $\mathrm{d} f / \mathrm{d} t(\mathrm{kHz} / \mathrm{s})$, excluding the quasiconstant $(\mathrm{d} f / \mathrm{d} t \sim 0)$ initial parts. When the frequency variation of a discrete emission is roughly approximated by a straight line, or sometimes by two straight lines, its or their gradients are plotted. A study by YosHIDA et al. (1983) on whistler-triggered VLF emissions indicated that the effective wave-particle interaction (leading to the generation of VLF emissions by whistlers) occurs in the off-equatorial region, and that the starting frequency of a triggered emission gives the location of the interaction region (YOSHIDA et $a l .$, 1983). The top panel of Fig. 3 indicates that $\mathrm{d} f / \mathrm{d} t$ of the majority of triggered emissions takes very large values, generally of the order of $10-20 \mathrm{kHz} / \mathrm{s}$ or more, and even the smallest one is $\sim 2.0 \mathrm{kHz} / \mathrm{s}$. These frequency drifts obtained for whistlertriggered emissions seem to be much larger than the normal values for chorus observed outside the plasmaspause by BURTIS and HELliwell (1976) and HAYAKAWA et al. (1984), but are nearly of the same order as for the discrete plasmaspheric emissions recently found by POULSEN and INAN (1988).

The bottom panel in Fig. 3 indicates the diurnal variation of dispersion of the causative whistler in order to estimate the latitude range where the relevant whistlertriggered VLF emissions are generated. When the dispersion is indicated by a vertical 

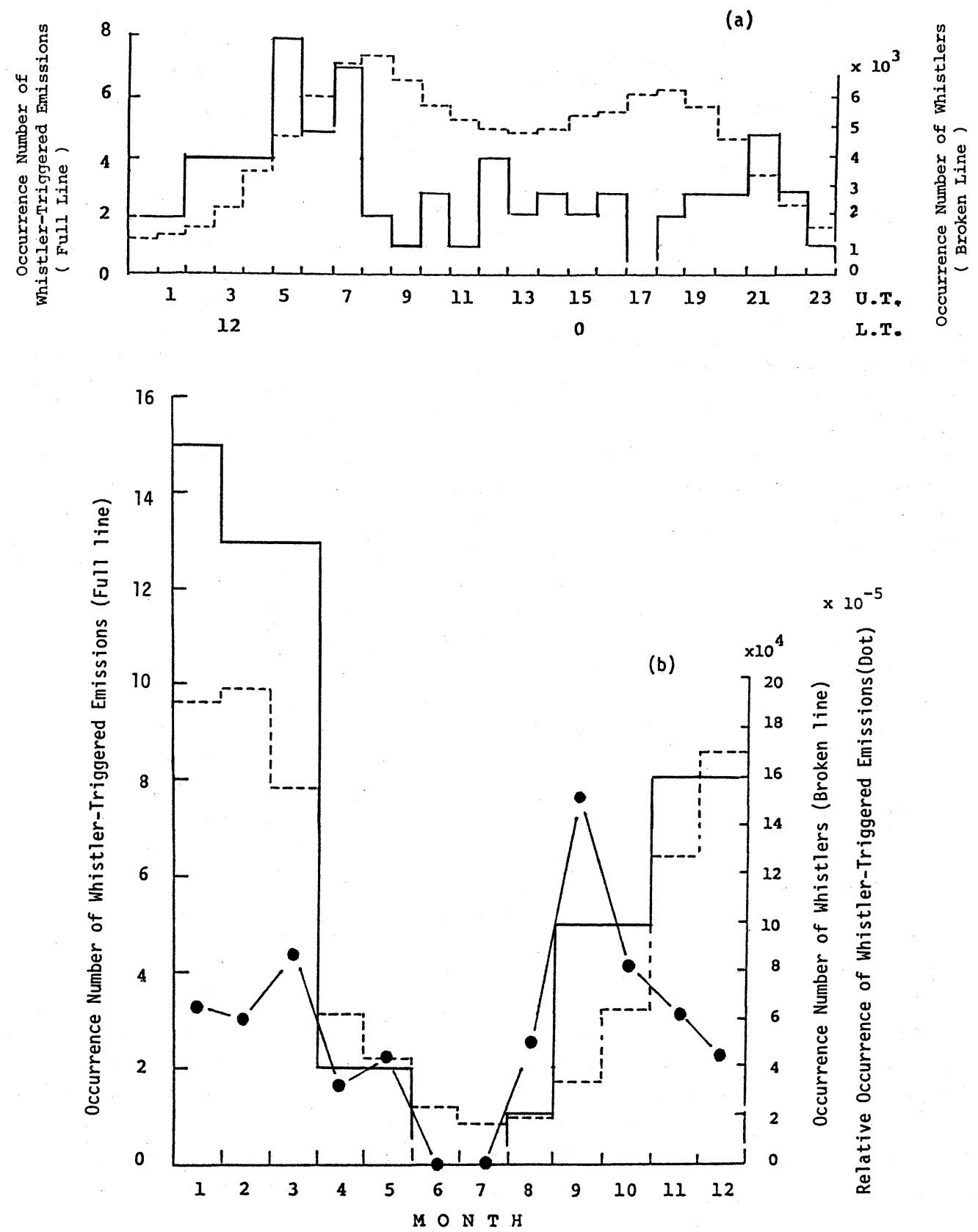

Fig. 1. (a) The diurnal variation of occurrence number of whistler-triggered VLF emissions (full line) and of the total number of whistlers recorded (broken line): L.T.=U.T. +9 h. (b) The seasonal variation of occurrence of whistler-triggered VLF emissions (full line), the corresponding seasonal variation of total number of whistlers recorded (broken line), and the relative occurrence of whistler-triggered VLF emissions (dot). (c) The relationship of the occurrence of whistler-triggered VLF emissions with $K p$ index at the time of emission observation (full line), and the percentage occurrence of number of times $K p$ is in each range (broken line). Dots indicate the relative occurrence probability of whistler-triggered VLF emissions. 


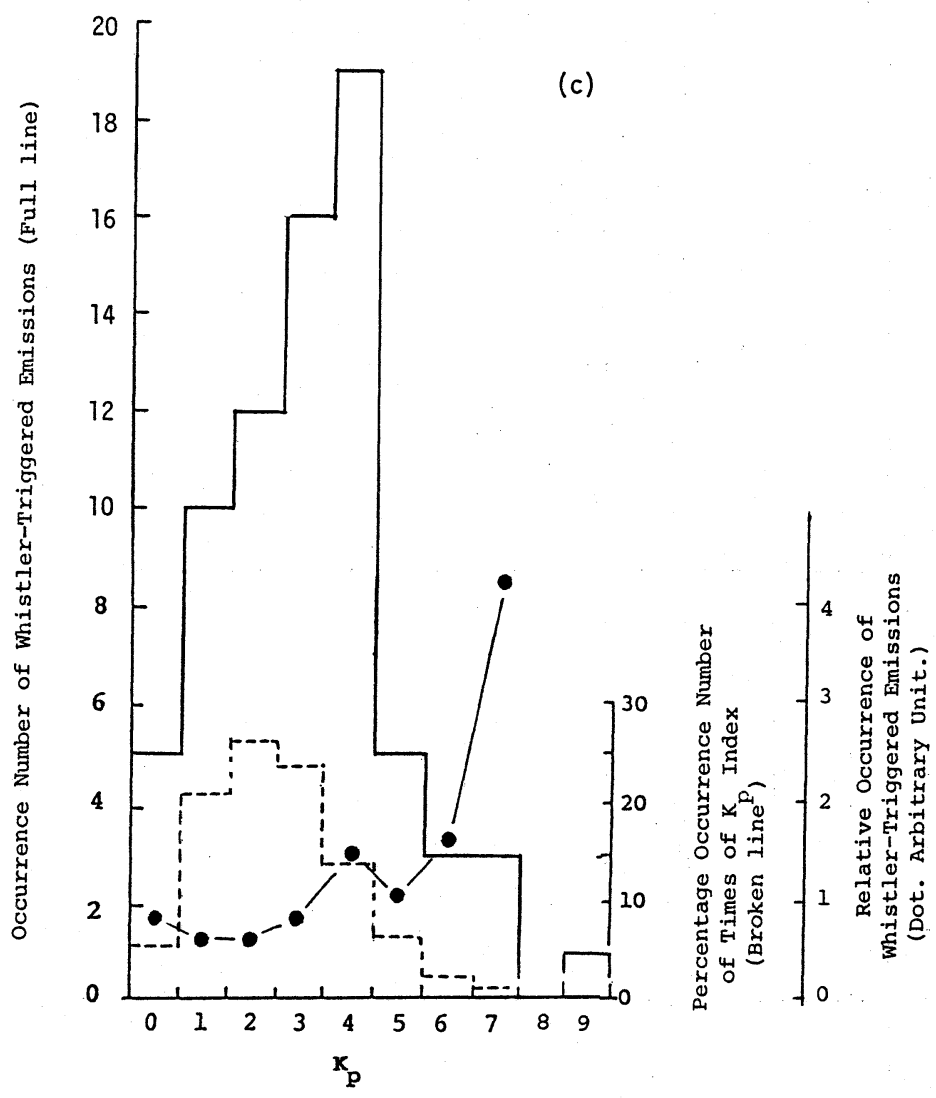

Fig. 1. (continued).

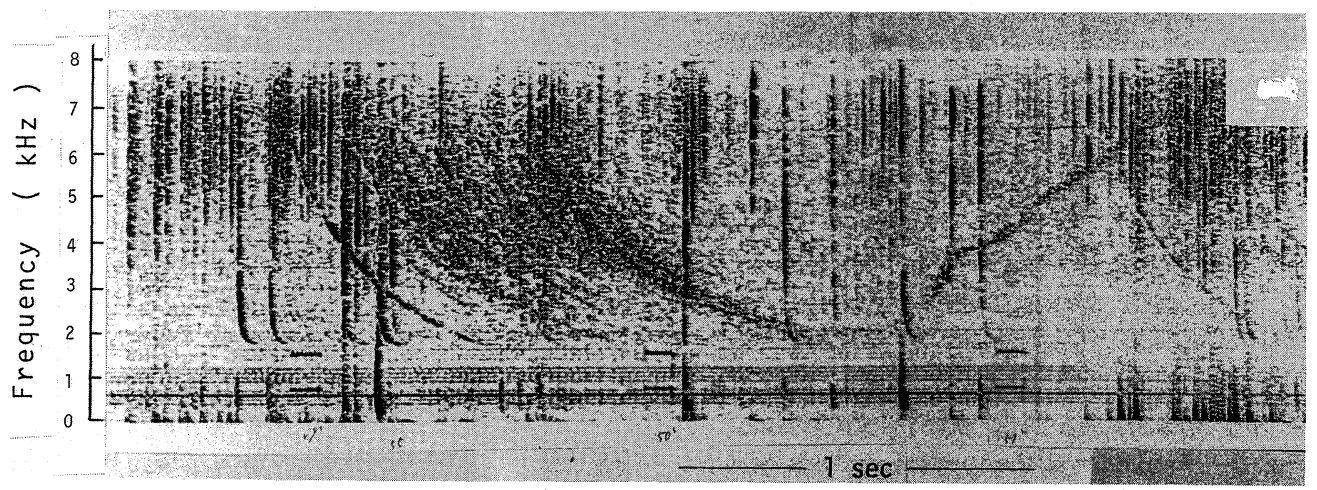

Fig. 2. An example of whistler-triggered VLF emissions at $10 \mathrm{~h} 50 \mathrm{~m}$ U.T. on November 6, 1978. There are a group of whistlers and the last whistler is found to trigger a rising-tone VLF emission from its extreme end of the spectrum. 

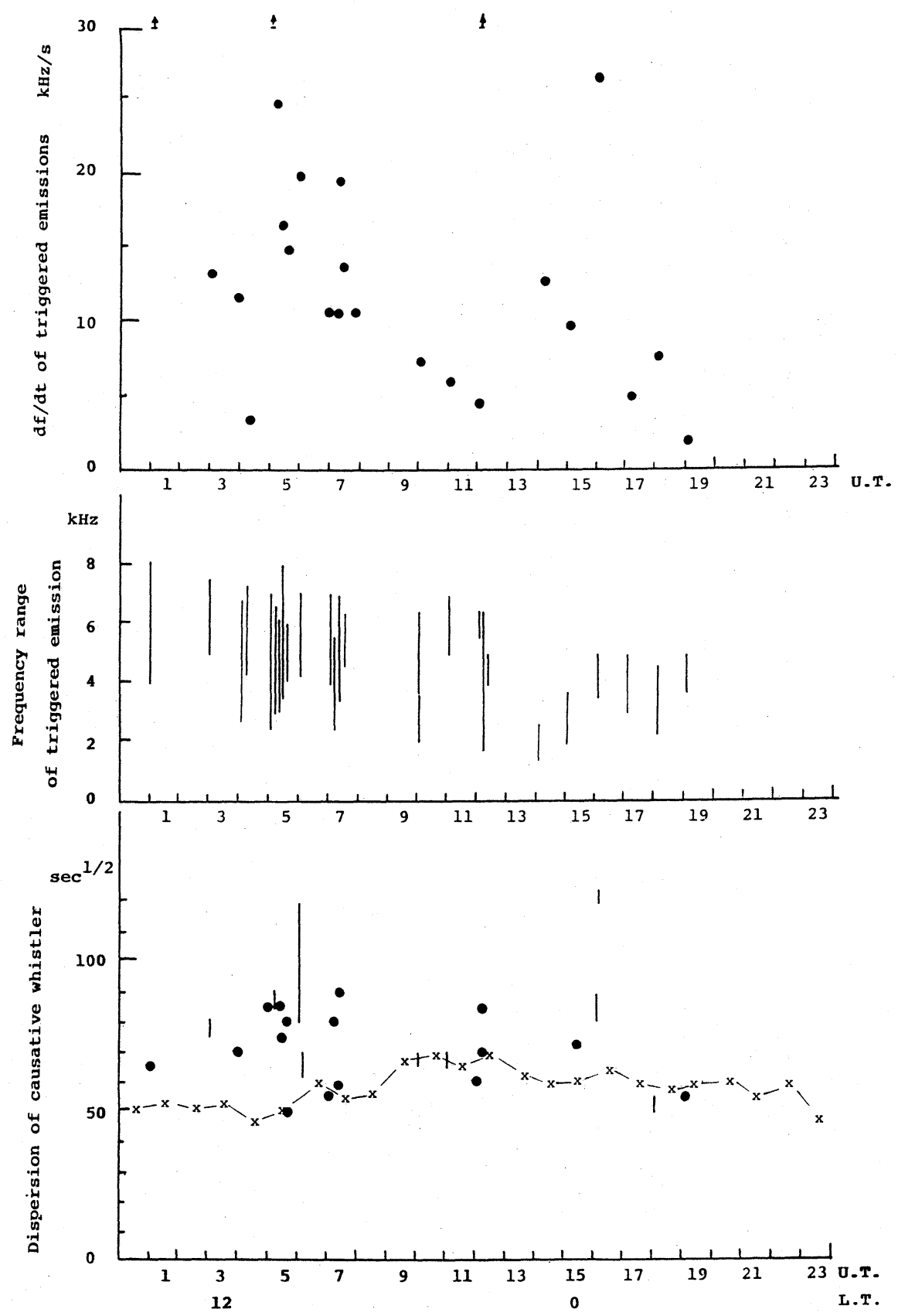

Fig. 3. Summary of the spectral analyses of whistler-triggered VLF emissions. The top and middle panels illustrate the diurnal variations of the frequency gradient, $\mathrm{d} f / \mathrm{d} t$ of triggered emissions (except the initial phase quasi-constant frequency components) (the arrows indicated upward at $\mathrm{d} f / \mathrm{d} t=30 \mathrm{kHz} / \mathrm{s}$ mean the $\mathrm{d} f / \mathrm{d} t$ being greater than $30 \mathrm{kHz} / \mathrm{s}$ ) and the frequency range occupied by the VLF emission (the lowest frequency roughly corresponds to the starting frequency of VLF emission), respectively. The bottom panel indicates the distribution of the dispersion of the causative whistler. Each dot corresponds to each event and a bar indicates the dispersion range of multi-path whistler. The curve connected by $\times$ is the diurnal variation of average dispersion. 
line, it means that we had multi-path whistlers whose dispersion range is plotted. The curve connected by $x$ is the diurnal variation of average dispersion of all ordinary whistlers observed at Moshiri from September to April (during which the whistlertriggered emissions are often observed as in Fig. 1(b)) over the relevant ten year span, which is in good agreement with the previous finding of KIMPARA (1962) who indicated a slight maximum in dispersion at U.T. $=12 \mathrm{~h}(\mathrm{~L} . \mathrm{T} .=21 \mathrm{~h})$. We here comment that the scatter of dispersion due to the solar-cycle variation is \pm less than $10 \%$ of the indicated average (HAYAKAWA et al., 1971). This curve is considered to correspond to the latitude of the observing station of Moshiri $(L=1.6)$, thus comparison of the dispersion of the causative whistler with the average dispersion, can be used to deduce the path latitude (or $L$ shell) of the generation region of whistler-triggered VLF emissions. We here comment that whistlers observed near Moshiri are found to be attributed to ducted propagation (HAYAKAWA et al., 1981) and whistlers at higher latitudes of $L>2$ are known to be ducted as well (HELLIWELL, 1965). There has been no previous work on the wave normal directions of triggered emissions, but it is very acceptable to presume that the associated emission has nearly the same wave normal angle as the causative whistler because both are considered to propagate in the same duct. Hence, the dispersion value can be used to deduce directly the $L$ shell on which a VLF emission is triggered by a whistler. The bottom panel in Fig. 3 implies that the dispersions of the causative whistlers of triggered emissions are roughly divided into two groups. One group consists of the dispersions located just around the average dispersion value, and the other is the group for which the dispersions are considerably larger than the average one. First, we deal with the latter group. The dispersion for this group is always higher than the average value by a range between 20 and $40 \mathrm{sec}^{1 / 2}$. By using ALLCOCK's (1960) empirical relationship $D=$ $2.2(\Lambda-12)$ where $D$ is dispersion $\left(\sec ^{1 / 2}\right)$ and $\Lambda$, the geomagnetic latitude (deg), the generation region of this group is likely to have been at a latitude higher than the station's latitude by $9^{\circ}$ to $18^{\circ}$, and correspondingly at $L$ ranges from 2.14 to 3.19. These $L$ values are known to fall in the region of the electron slot. Next, we discuss the first group. The generation region of whistler-triggered VLF emissions for this group is apparently around $L=1.6$ (and sometimes slightly below $L=1.6$ ) in the inner radiation belt.

\section{Summary and Discussion}

The statistical analyses of whistler-triggered VLF emissions during the ten-year span from 1976 to 1985, yielded the following findings.

1) A simple ratio of occurrence number of whistler-triggered emissions to the total whistler rate provides an occurrence probability of whistler-triggered emissions of $\sim 3 \times 10^{-4}$.

2) There is no clear tendency for whistler-triggered VLF emissions to occur at a particular L.T., and two equinoctial maxima in occurrence probability are recognized.

3) There seems to exist a general tendency for the occurrence probability to increase with increasing $K p$ in the range from 3 to 7 .

4) The occurrence $L$ shell of whistler-triggered emissions is grouped into two ranges; one is $L=2.1$ to 3.2 in the electron slot region and another just around $L=1.6$ in the inner radiation belt.

5) The spectral feature of the whistler-triggered emission is such that the initial part of the emission starting from the whistler spectrum is nearly at a constant frequency and 
the subsequent change is very dynamic with $\mathrm{d} f / \mathrm{d} t$, mainly in a range from 10 to $20 \mathrm{kHz} / \mathrm{s}$.

The occurrence latitudes of whistler-triggered emissions were estimated from the present study to be located in the electron slot $(2.14<L<3.19)$ as summarized in Item (4), which agrees with the corresponding $L$ range of lightning-induced particle precipitations by Helliwell et al. (1973), CARPENTER and LABELLE (1982), LEYSER et al. (1984), VOSS et al. (1984), INAN et al. (1985), INAN and CARPENTER (1987) and CARPENTER and INAN (1987). This preferred $L$ shell is likely to be consistent with the theoretical prediction by CHANG and INAN (1985). They obtained the result that for a higher energy (40-300 keV) electron precipitation due to gyroresonance, there exists an inner magnetospheric region $(2<L<3)$ where the level of whistler-induced precipitation can be expected to be comparatively high. Correspondingly, we would expect effective wave-particle interaction in this $L$ range, leading to the enhanced occurrence of whistler-triggered VLF emissions. Of course, none of the pitch angle scattering models, as mentioned above, considered the wave growth. The conditions necessary for emissions are likely to be much more difficult to attain, which may be reflected in the low number of occurrences (Item (1)). The present study (Item (4)) implied an additional occurrence region of whistlertriggered emissions around $L=1.6$ corresponding to the location of the inner radiation belt. Our finding on the wave aspect of gyroresonance is likely to be supported by a recent study by INAN et al. (1988) who found that the region of whistler-induced precipitation extends even to the inner radiation belt $(L \leq 1.8)$. Furthermore, NAGATA et al. (1988) recently found, based on data from the EXOS-C satellite, the presence of a preferred $L$ range ( $L=1.6-1.8$ ) of the $0.19-3.2 \mathrm{MeV}$ electron precipitation. The starting frequency of triggered VLF emissions is, in many occasions, in a range from 2.0 to 4.0 $\mathrm{kHz}$ (middle panel of Fig. 3) and so the gyroresonance electron energies at such frequencies for the causative whistlers are in the $\mathrm{MeV}$ range in the inner radiation belt.

Item (2) indicates that whistler-triggered VLF emissions which occurred over the whole L.T. when whistlers were recorded. No clear tendency for whistler-triggered emissions was again in agreement with the corresponding diurnal variation of whistlerinduced particle precipitation by CARPENTER and INAN (1987). The wide L.T. distribution of event activity suggested that electrons drifting toward the dawn sector from injection points on the nightside were not a controlling factor whereas HAYAKAWA (1989) showed that the dawn is a preferred sector for substorm-associated VLF/ELF emissions.

As found in HAYAKAWA and OHTSU (1973), some portions of the whistlers observed from Moshiri are those which existed the ionosphere at a latitude much lower than the station's latitude. Furthermore, our station, Moshiri, is not located in a very suitable place for the observation of phenomena of whistler-triggered emissions generated at $2<L<3$ because of an attenuation from propagation in the Earth-ionosphere waveguide. When we consider these two factors, the occurrence probability of triggering emissions by whistlers of $\sim 3 \times 10^{-4}$ (Item (1)) may increase to a considerable extent. The joint influences of the activity of lightning sources and particle dynamics in the magnetosphere remain to be assessed.

The occurrence probability of whistler-triggered emissions increased with increasing $K p$ in the range from 3 to 7 as given by Item (3), which is found to be in a sharp contrast with the corresponding dependence of whistler occurrence number upon $K p$. The obtained tendency is in agreement with the corresponding behaviour of lightning-induced precipitation reported by CARPENTER and LABELLE (1982) and LEYSER et al. (1984). 
This effect is easily explained by the enhanced particle flux of resonance electrons during higher geomagnetic activities.

The seasonal variation is noticeable as shown in Item (2). The austral summer drop in the activity of whistler-triggered emissions can be partly understood as being due to reduced lightning source activity in the conjugate region, but the reduced activity in an austral winter is difficult to understand. A factor which may be related to it, is the change in energetic electron flux levels established at $L=2-3$ within the plasmasphere in the aftermath of large magnetic storms. There is a semiannual variation in magnetic activity (e.g. RUSSELl and MCPHERRON, 1973). GREEN (1984) found that this variation is dominated by the semiannual modulation, with equinoctial maxima, of the occurrence of large storms. Another influential factor may be the semiannual variation of the electron density in the magnetosphere. A semiannual variation of the dispersion of whistlers was clearly noticed by HAYAKAWA et al. (1971) from Moshiri and by CORCUFF (1965) from Poitiers $(L=1.90)$, in which the equinoctial maxima were apparent. Enhanced maximum in the electron density near the equator (correspondingly even at slightly off-equatorial region where whistler-triggered emissions are generated (YosHIDA et al., 1983)) is expected in the equinoxes. This means that the energy of resonant electrons for whistlertriggered emissions is lowered for more enhanced density (e.g. BRICE, 1964) during equinoxes, which leads to enhanced wave-particle interaction. Hence, the above two factors are considered to be in operation combinationally for the equinoctial maxima of the occurrence probability of whistler-triggered emissions, and they are consistent with the gyroresonance interaction between whistler waves and energetic electrons.

The detailed characteristics of spectral shapes of whistler-triggered emissions are summarized as Item (5). A further study of these properties is beyond the scope of this paper, and will be published elsewhere.

The author is grateful to his colleagues and members of VLF group of Nagoya University for their useful discussion and also to Dr. S. Shimakura of Chiba University, Prof. H. Kikuchi of Nihon University and the unknown referees for their useful comments. Thanks are also due to Mrs. H. Yamaguchi of VLF group for her careful data analyses. Finally, the continuous encouragement of Dr. A. Kimpara, Emeritus Professor of Nagoya University is greatly appreciated.

\section{REFERENCES}

Allcock, G. McK., IGY whistler results, Paper presented at 13th General Assembly of URSI, London, 1960. Allcock, G. McK., Whistler propagation and geomagnetic activity, J. Inst. Telecomm. Eng. New Delhi, 12, $158-168,1966$.

BRICE, N. M., Discrete VLF emissions from the upper atmosphere, Tech. Rept. No. SEL64-088, Radioscience Lab., Stanford Univ., Stanford, CA, 1964.

Burtis, W. J. and R. A. Helliwell, Magnetospheric chorus: Occurrence patterns and normalized frequency, Planet. Space Sci., 24, 1007-1024, 1976.

CARPENTER, D. L. and U. S. InAN, Seasonal, latitudinal and diurnal distributions of whistler-induced electron precipitation events, J. Geophys. Res., 92, 3429-3435, 1987.

CARPENTER, D. L. and J. W. LABELle, A study of whistlers correlated with bursts of electron precipitation near $L=2$, J. Geophys. Res., 87, 4427-4434, 1982.

Chang, H. C. and U. S. INAN, Lightning-induced energetic electron precipitation from the magnetosphere, $J$. Geophys. Res., 90, 1531-1541, 1985.

Corcuff, Y., Étude de la magnétosphère au moyen des sifflements radioelectriques, Thèse, Université de Poitiers, 1965. 
Green, C. A., The semiannual variation in the magnetic activity indices Aa and Ap, Planet. Space Sci., 32, 297-305, 1984.

HayaKawa, M., Further study of the frequency drift of dawnside mid-latitude VLF emissions associated with magnetic disturbances, Planet. Space Sci., 37, 269-276, 1989.

Hayakawa, M. and J. OHTsu, Ducted propagation of low latitude whistlers deduced from the simultaneous observations at multi-stations, J. Atmos. Terr. Phys., 35, 1685-1697, 1973.

Hayakawa, M., J. Ohtsu, and A. IwaI, Characteristics of dispersion and occurrence rate of whistlers at low latitudes during one solar cycle, J. Geomag. Geoelectr., 23, 187-201, 1971.

Hayakawa, M., Y. TANAKA, and J. Ohtsu, On the morphologies of auroral and low latitude VLF 'hiss', $J$. Atmos. Terr. Phys., 37, 517-529, 1975.

Hayakawa, M., T. OKaDA, and A. IwaI, Direction finding of medium latitude whistlers and their propagation characteristics, J. Geophys. Res., 86, 6939-6946, 1981.

Hayakawa, M., Y. Yamanaka, M. Parrot, and F. Lefeuvre, The wave normals of magnetospheric chorus emissions observed on board GEOS-2, J. Geophys. Res., 89, 2811-2821, 1984.

Hayakawa, M., T. OKada, and Y. TANakA, Morphological characteristics and the polarization of plasmaspheric ELF hiss observed at Moshiri (L 1.6), J. Geophys. Res., 90, 5133-5140, 1985.

Helliwell, R. A., Whistlers and Related Ionospheric Phenomena, 349 pp., Stanford Univ. Press, Stanford, CA, 1965.

Helliwell, R. A., J. P. Katsufrakis, and M. L. Trimpi, Whistler-induced amplitude perturbations in VLF propagation, J. Geophys. Res., 78, 4679-4688, 1973.

INAN, U. S. and D. L. CARPENTER, Lightning-induced precipitation events observed at $L=2.4$ as phase and amplitude perturbations on subionospheric VLF signals, J. Geophys. Res., 92, 3293-3303, 1987.

Inan, U. S., D. L. Carpenter, R. A. Helliwell, and J. P. Katsufrakis, Subionospheric VLF/LF phase perturbations by lightning-induced particle precipitation, J. Geophys. Res., 90, 7457-7469, 1985.

Inan, U. S., W. C. Burgess, T. G. Wolf, D. C. Shafer, and R. E. Orville, Lightning-associated precipitation of $\mathrm{MeV}$ electrons from the inner radiation belt, Geophys. Res. Lett., 15, 172-175, 1988.

Kimpara, A., On some remarkable characteristics on whistler atmospherics, Proc. Res. Inst. Atmospherics, Nagoya Univ., 7, 40-57, 1960.

Kimpara, A., Some characteristics of the dispersion of whistlers, Proc. Res. Inst. Atmospherics, Nagoya Univ., 9, 5-17, 1962.

LEYSER, T. B., U. S. InAN, D. L. CARPENTER, and M. L. TRIMPI, Diurnal variation of burst precipitation effects on subionospheric VLF/LF signal propagation near L=2, J. Geophys. Res., 89, 9139-9143, 1984.

Nagata, K., T. Kohno, H. Murakami, A. Nakamoto, N. Hasebe, J. Kikuchi, and T. Dote, Electron $(0.19-3.2 \mathrm{MeV})$ and proton $(0.58-35 \mathrm{MeV})$ precipitations observed by OHZORA satellite at low latitude zones L=1.6-1.8, Planet. Space Sci., 36, 591-606, 1988.

Poulsen, W. L. and U. S. InAN, Satellite observations of a new type of discrete VLF emissions at $L<4, J$. Geophys. Res., 93, 1817-1838, 1988.

Russell, C. T. and R. L. MCPherron, Semiannual variation of geomagnetic activity, J. Geophys. Res., 78, 92-108, 1973.

Voss, H. D., W. L. Imhof, J. Mobilia, E. E. Gaines, M. Walt, U. S. Inan, R. A. Helliwell, D. L. Carpenter, J. P. Katsufrakis, and H. C. Chang, Lightning-induced electron precipitation, Nature, 312, 740-742, 1984.

Yoshida, T., J. OHTSU, and M. Hayakawa, A study of the mechanism of whistler-triggered VLF emissions, $J$. Geophys., 53, 59-72, 1983. 